Correction

\title{
Correction: Wen et al. Fabrication of Dense Gadolinia-Doped Ceria Coatings via Very-Low-Pressure Plasma Spray and Plasma Spray-Physical Vapor Deposition Process. Coatings 2019, 9, 717
}

Jing Wen ${ }^{1,2,+}$, Chen Song ${ }^{2,3, *,+}$, Taikai Liu ${ }^{2}$, Ziqian Deng ${ }^{2}$, Shaopeng Niu ${ }^{2}$, Yapeng Zhang ${ }^{2}$, Libin Liu ${ }^{1}$ and Min Liu ${ }^{2}$

1 School of Materials Science and Engineering, Central South University, Changsha 410083, China; 173114197@csu.edu.cn (J.W.); lbliu@csu.edu.cn (L.L.)

2 Guangdong Institute of New Materials, National Engineering Laboratory for Modern Materials Surface Engineering Technology, the Key Lab of Guangdong for Modern Surface Engineering Technology, Guangzhou 510651, China; liutaikai@gdinm.com (T.L.); dengziqian@gdinm.com (Z.D.); niushaopeng@gdinm.com (S.N.); zhangyapeng@gdinm.com (Y.Z.); liumin@gdas.gd.cn (M.L.)

3 School of Environment and Energy, South China University of Technology, Guangzhou 510006, China

* Correspondence: songchen@gdinm.com

+ Co-first author.

Received: 7 February 2020; Accepted: 13 February 2020; Published: 20 March 2020

The author wishes to make the following correction to this paper [1]. Due to mislabeling, replace:

Table 2. Parameters of the very-low-pressure plasma spray (VLPPS) and plasma spray-physical vapor deposition (PS-PVD).

\begin{tabular}{ccc}
\hline Parameters & VLPPS Coating & PS-PVD Coating \\
\hline Pressure $(\mathrm{Pa})$ & 150 & 150 \\
Current $(\mathrm{A})$ & 720 & 2600 \\
Torch & $\mathrm{F} 4-\mathrm{VB}$ & O3CP \\
Power $(\mathrm{kW})$ & 53 & 126 \\
Plasma gas $(\mathrm{Ar})(\mathrm{slpm})^{\mathrm{a}}$ & 60 & 30 \\
Plasma gas $(\mathrm{He})(\mathrm{slpm})^{\mathrm{a}}$ & - & 90 \\
${\text { Plasma gas }\left(\mathrm{H}_{2}\right)(\mathrm{slpm})^{\mathrm{a}}}^{\mathrm{a}}$ & 12 & - \\
Powder feed rate $\left(\mathrm{g} \cdot \mathrm{min}^{-1}\right)$ & 2 & 10 \\
Spraying distance $(\mathrm{mm})$ & 150 & 800 \\
Substrate temperature $\left({ }^{\circ} \mathrm{C}\right)$ & $900-1000$ & $800-900$ \\
Torch speed $(\mathrm{mm} / \mathrm{s})$ & 300 & 500 \\
\hline
\end{tabular}

with 
Table 2. Parameters of the very-low-pressure plasma spray (VLPPS) and plasma spray-physical vapor deposition (PS-PVD).

\begin{tabular}{ccc}
\hline Parameters & VLPPS Coating & PS-PVD Coating \\
\hline Pressure $(\mathrm{Pa})$ & 150 & 150 \\
Current $(\mathrm{A})$ & 720 & 2600 \\
Torch & $\mathrm{F} 4-\mathrm{VB}$ & O3CP \\
Power $(\mathrm{kW})$ & 53 & 126 \\
Plasma gas $(\mathrm{Ar})(\mathrm{slpm})^{\mathrm{a}}$ & 60 & 90 \\
Plasma gas $(\mathrm{He})(\mathrm{slpm})^{\mathrm{a}}$ & - & 30 \\
Plasma gas $\left(\mathrm{H}_{2}\right)(\mathrm{slpm})^{\mathrm{a}}$ & 12 & - \\
Powder feed rate $\left(\mathrm{g} \cdot \mathrm{min}^{-1}\right)$ & 2 & 10 \\
Spraying distance $(\mathrm{mm})$ & 250 & 800 \\
Substrate temperature $\left({ }^{\circ} \mathrm{C}\right)$ & $900-1000$ & $800-900$ \\
Torch speed $(\mathrm{mm} / \mathrm{s})$ & 300 & 500 \\
\hline
\end{tabular}

The revised numbers are marked in red in the corrected table.

The authors would like to apologize for any inconvenience caused to the readers by these changes.

\section{Reference}

1. Wen, J.; Song, C.; Liu, T.; Deng, Z.; Niu, S.; Zhang, Y.; Liu, L.; Liu, M. Fabrication of Dense Gadolinia-Doped Ceria Coatings via Very-Low-Pressure Plasma Spray and Plasma Spray-Physical Vapor Deposition Process. Coatings 2019, 9, 717. [CrossRef]

(C) 2020 by the authors. Licensee MDPI, Basel, Switzerland. This article is an open access article distributed under the terms and conditions of the Creative Commons Attribution (CC BY) license (http://creativecommons.org/licenses/by/4.0/). 